\title{
An Inverse Thermogelling Bioink Based on an ABA-Type Poly(2-oxazoline) Amphiphile
}

\section{Hahn, Lukas}

2021-07-12

Hahn , L , Karakaya , E , Zorn , T, Sochor , B , Maier , M , Stahlhut , P , Forster , S , Fischer , K, Seiffert , S , Pöppler , A-C , Detsch , R \& Luxenhofer , R 2021 , ' An Inverse

Thermogelling Bioink Based on an ABA-Type Poly(2-oxazoline) Amphiphile ' ,

Biomacromolecules, vol. 22 , no. 7 , pp. 3017-3027 . https://doi.org/10.1021/acs.biomac.1c00427

http://hdl.handle.net/10138/333369

https://doi.org/10.1021/acs.biomac.1c00427

cc_by

publishedVersion

Downloaded from Helda, University of Helsinki institutional repository.

This is an electronic reprint of the original article.

This reprint may differ from the original in pagination and typographic detail.

Please cite the original version. 


\section{An Inverse Thermogelling Bioink Based on an ABA-Type Poly(2- oxazoline) Amphiphile}

Lukas Hahn, Emine Karakaya, Theresa Zorn, Benedikt Sochor, Matthias Maier, Philipp Stahlhut, Stefan Forster, Karl Fischer, Sebastian Seiffert, Ann-Christin Pöppler, Rainer Detsch, and Robert Luxenhofer*

Cite This: Biomacromolecules 2021, 22, 3017-3027

Read Online

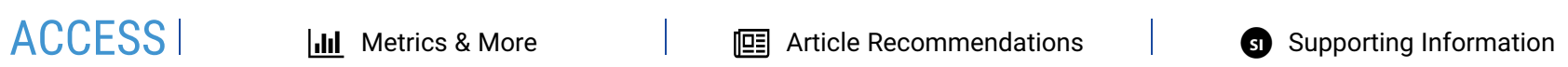

ABSTRACT: Hydrogels are key components in several biomedical research areas such as drug delivery, tissue engineering, and biofabrication. Here, a novel ABA-type triblock copolymer comprising poly(2-methyl-2-oxazoline) as the hydrophilic A blocks and poly(2-phenethyl-2oxazoline) as the aromatic and hydrophobic B block is introduced. Above the critical micelle concentration, the polymer self-assembles into small spherical polymer micelles with a hydrodynamic radius of approx $8-8.5 \mathrm{~nm}$. Interestingly, this specific combination of hydrophilic and hydrophobic aromatic moieties leads to rapid thermoresponsive inverse gelation at polymer

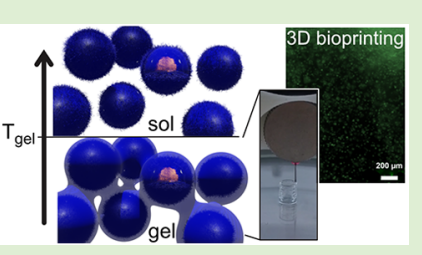
concentrations above a critical gelation concentration (20 wt \%) into a macroporous hydrogel of densely packed micelles. This hydrogel exhibited pronounced viscoelastic solid-like properties, as well as extensive shear-thinning, rapid structure recovery, and good strain resistance properties. Excellent 3D-printability of the hydrogel at lower temperature opens a wide range of different applications, for example, in the field of biofabrication. In preliminary bioprinting experiments using NIH 3T3 cells, excellent cell viabilities of more than $95 \%$ were achieved. The particularly interesting feature of this novel material is that it can be used as a printing support in hybrid bioink systems and sacrificial bioink due to rapid dissolution at physiological conditions.

\section{INTRODUCTION}

Thermosensitive water-soluble polymers undergo phase separation due to temperature changes. Phase separation upon heating is characterized by a lower critical solution temperature (LCST). ${ }^{1}$ In contrast, in a polymer, which exhibits an upper critical solution temperature (UCST), phase separation takes place upon cooling. ${ }^{2-7}$ Exemplarily, Seuring et al. reported and characterized the UCST-type phase separation properties of a nonionic homopolymer poly $(N$-acryloyl glycinamide $)$ in pure water. ${ }^{8}$ In a more recent study poly(acrylic acid) was copolymerized with acrylonitrile resulting in an UCST-type copolymer combining the driving forces of hydrogen bonding and hydrophobic interactions. ${ }^{9}$ In some cases hydrogel formation instead of phase separation/precipitation is observed. Over the last decades, most described systems in the literature are based on synthetic or natural water-soluble polymers exhibiting thermosensitive gelation upon heating. ${ }^{0-15}$ By introducing thermoresponsive units into the polymer structure, gelation can be induced at room or elevated temperatures as was described many times. ${ }^{16,17}$ Only a few examples can be found in the literature where the gelation occurred upon cooling the sample below a critical temperature (inverse gelation). Particularly prominent examples are natural biopolymers like gelatin, agarose, and pectin/chitosan systems. ${ }^{18,19}$ In 1998 Yoshioka et al. reported a gelatin- $g$-poly $(N$-isopropylacrylamide) graft copolymer which undergoes thermogelation (above $34^{\circ} \mathrm{C}$ ) and inverse thermogelation (below $10^{\circ} \mathrm{C}$ ) at $5 \mathrm{wt} \% .^{20} \mathrm{In}$ addition to rheological investigations, the hypothetical use of this system in smart 3D cell culture was discussed. In 2016, an inverse thermogelling ABA-type block copolymer comprising a poly(poly(ethylene glycol) methyl ether methacrylate) middle block and thermosensitive poly(acrylamide-co-acrylonitrile) $(\mathrm{P}(\mathrm{AAm}-\mathrm{co}-\mathrm{AN}))$ outer blocks was described. ${ }^{21}$ The temperature-dependent sol/gel properties were investigated in the temperature range of $8-80^{\circ} \mathrm{C}$ and low polymer concentrations of 3 and $5 \mathrm{wt} \%$. At $8{ }^{\circ} \mathrm{C}$, a clear hydrogel was obtained. The sol/ gel transition could be modulated by the polymer composition. By modifying a high molecular weight precursor copolymer comprising $N, N$-dimethylacrylamide and acrylic acid with hydrophobic moieties of $n$-dodecyl amine, an inverse thermogelling hydrogel could be realized. ${ }^{22}$ At $0{ }^{\circ} \mathrm{C}$, a viscosity of approx. $1 \mathrm{kPas}$ was obtained by varying the sol/gel temperature with different polymer concentrations. However, no defined application as biomaterials has been described here either. Inspired by the water transfer of natural mimosa, Chen and co-workers established a thermoresponsive bilayer system in $2018 .^{23}$ One part of the system was an inverse thermogelling hydrogel formed by poly(acrylic acid-co-acrylamide) (PAAc-coPAAm) layers. The system was discussed as an actuator for the

Received: April 1, 2021

Revised: $\quad$ May 14, 2021

Published: June 8, 2021 
Scheme 1. Synthesis Route ${ }^{a}$
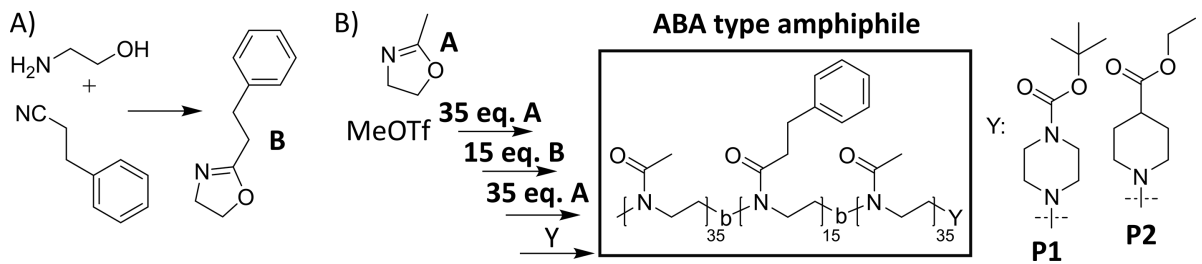

${ }^{a}$ (A) Synthesis of PhenEtOx (B) adapted from Witte and Seeliger. ${ }^{41}$ (B) Synthesis of the ABA-type amphiphile by living cationic ring-opening polymerization of the hydrophilic $\mathrm{MeOx}(\mathrm{A})$ and the hydrophobic and aromatic PhenEtOx (B) using methyl triflate (MeOTf) as an initiator. To introduce the terminal Y-functionality, BOC-Pip (P1) and EIP (P2) were used as terminating reagents, respectively.

fabrication of intelligent soft materials for bioinspired applications. Nevertheless, the embedding of living cells seems to be challenging as the cells sediment before polymerization and the actual hydrogel formation is performed at $50{ }^{\circ} \mathrm{C}$ under ultraviolet light (UV) exposure. The combination of such conditions can be expected to cause significant cytotoxicity. In 2019, Hua et al. reported a multi-thermoresponsive hydrogel system, which offers the possibility of 3D shape transition upon cooling in water and oil systems. ${ }^{24}$ In the last years, the interest in biofabrication, especially for tissue engineering and regenerative medicine has been rapidly growing into a promising interdisciplinary research field in its own right. ${ }^{25}$ One major additive manufacturing technique used in the field is extrusionprinting of bioinks. ${ }^{26}$ Often a hydrogel formulation, which enables printability and cell survival during the printing process, is preferentially used. ${ }^{27}$ To date, most commonly, hydrogels based on natural biopolymers, such as gelatin, alginate, and hyaluronic acid, are used due to their generally good cytocompatibility. ${ }^{28}$ However, such systems based on biopolymers can also have limitations such as less-than-ideal printability and often considerable batch-to-batch variations, especially with respect to rheological properties. The synthetic polymer platform formed by cyclic imino ethers, in particular poly(2-substituted-2-oxazoline)s (POx) and poly(2-substituted-5,6-dihydro-4H-1,3-oxazine)s (poly(2-oxazine)s, POzi), was investigated for decades as biomaterials in different applications due to good cytocompatibility and chemical versatility. ${ }^{29-32} \mathrm{POx} / \mathrm{POzi}$-based systems were investigated as thermoresponsive materials ${ }^{32}$ for biomedical applications ${ }^{33,34}$ and drug delivery ${ }^{35}$ approaches. However, surprisingly few reports can be found that show thermogelation of pure POx/ POzi polymers in water. In 2017, Lorson et al. established a thermogelling cytocompatible and printable supramolecular hydrogel based on POx/POzi diblock copolymers. ${ }^{36}$ The system comprised a hydrophilic poly (2-methyl-2-oxazoline) (PMeOx) block and a thermoresponsive poly(2-n-propyl-2-oxazine) (PnPrOzi) block of similar block length. Another thermogelling hydrogel was described by Hoogenboom and Monnery. ${ }^{37} \mathrm{~A}$ $\mathrm{BAB}$ triblock copolymer bearing poly(2-n-propyl-2-oxazoline) ( $\mathrm{P} n \mathrm{PrOx})(\mathrm{B})$ and hydrophilic poly(2-ethyl-2-oxazoline) blocks (A) showed sol/gel transitions upon heating, but extremely high degrees of polymerization were required. In 2019, Lübtow et al. described a different, much shorter ABA triblock copolymer, which forms stable, but relatively weak and barely printable, thermogelling hydrogels. ${ }^{38}$ Very recently, the first inverse thermogelling hydrogel formed by $\mathrm{POx} / \mathrm{POzi}$-based block copolymers was described. ${ }^{39}$ Here, the ABA triblock copolymer comprising the hydrophilic $\mathrm{PMeOx}(\mathrm{A})$ and aromatic hydrophobic poly(2-phenyl-2-oxazine) (PPheOzi, B) blocks undergoes gelation at lower temperatures. The stable hydrogel was formed by entangled self-assembled wormlike micelles, which form from spherical micelles upon cooling, leading to gelation. A potential application of such system as a biomaterial ink was mentioned. The limiting factor for using this system as a component in a bioink formulation is its gelation kinetics. Upon cooling, it takes about $1 \mathrm{~h}$ to form a hydrogel suitable for printing, which is a rather long time if viable cells are to be embedded. Very recently, a novel gelation mechanism driven by interactions between the hydrophilic PMeOx blocks and the aromatic PPheOzi blocks was suggested for this system. ${ }^{40}$ Due to this interaction, the volume fraction of the hydrophilic to hydrophobic domain changes, leading to the morphology switch. Interestingly, polymers containing very similar 2-phenyland 2-benzyl-2-oxazoline and 2-benzyl-2-oxazine did not undergo this gelation. Here, a further structural variation and a new $\mathrm{ABA}$ triblock copolymer containing aromatic poly(2phenethyl-2-oxazoline) (PPhenEtOx, B) is described, which can be seen as a higher homologue or structural isomer of previously studied polymers, and it again undergoes inverse thermogelation in aqueous solution. In contrast to previously studied material, a rapid thermoresponsive sol/gel transition was observed, which facilitates its use as a bioink component, as was demonstrated. The additional two $\mathrm{CH}_{2}$-groups in the sidechain as well as the different backbones compared to the previously described inverse hydrogel platform, leads to distinct differences in the gelation mechanism as in the present case, the size and morphology of the polymer self-assemblies do not change with temperature.

\section{EXPERIMENTAL SECTION}

In general, all substances and reagents for the monomer synthesis and polymerization were purchased from Sigma-Aldrich (Steinheim, Germany) and TCI-chemicals (Eschborn, Germany) and were used as-received without further purification unless otherwise stated. For polymerization, all substances were refluxed over $\mathrm{CaH}_{2}$ for several hours and distilled prior usage. The solvent benzonitrile $(\mathrm{PhCN})$ was dried over phosphorus pentoxide.

The monomer synthesis of 2-phenethyl-2-oxazoline (Schemes 1A and S1) was carried out as described by Witte and Seeliger. ${ }^{41}$ For the reaction, 1 equiv of 3-phenylpropionitrile, 1.2 equiv of amino-ethanol, and catalytic amounts of zinc acetate dihydrate were added to a argonflushed flask and heated to $130^{\circ} \mathrm{C}$ under reflux for several days until the color of the reaction mixture turned brown. Reaction progress was controlled by ${ }^{1} \mathrm{H}$ NMR spectroscopy. After completion, the mixture was dissolved in dichloromethane and washed three times with $\mathrm{H}_{2} \mathrm{O}$. The organic phase was dried with $\mathrm{MgSO}_{4}$ and concentrated. The raw product was refluxed with $\mathrm{CaH}_{2}$ and purified via vacuum distillation under an argon atmosphere yielding a colorless liquid. The resulting compound 2-phenethyl-2-oxazoline was characterized via refractive index, gas chromatography-electrospray ionization-mass spectrometry (GC-ESI-MS) analysis, and ${ }^{1} \mathrm{H}$ and ${ }^{13} \mathrm{C}$ nuclear magnetic resonance (NMR) spectroscopy (see the Supporting Information). 
Refractive index measurements of the synthesized monomer 2phenethyl-2-oxazoline was performed on a RFM 870 refractometer from Bellingham + Stanley at $20^{\circ} \mathrm{C}$ (Farnborough, England).

The monomer was further analyzed via mass spectrometry using an Agilent 5977B MDS system coupled with a gas chromatography system Agilent 7820A. The GC system was equipped with an Agilent 19091S433UI HP-5 ms ultrainert column $(30 \mathrm{~m} \times 250 \mu \mathrm{m} \times 0.25 \mu \mathrm{m})$. The temperature gradient was set from 40 to $300{ }^{\circ} \mathrm{C}$ with a constant heat rate of $15{ }^{\circ} \mathrm{C} / \mathrm{min}$ and a constant flow of $1 \mathrm{~mL} / \mathrm{min}$.

The ABA-type copolymers were synthesized following a general procedure based on previous reports (detailed description in the Supporting Information).42,43

NMR was performed on a Bruker Fourier $300\left({ }^{1} \mathrm{H}: 300.12 \mathrm{MHz}\right)$ spectrometer at $298 \mathrm{~K}$ from Bruker BioSpin (Rheinstetten, Germany) and calibrated using the residual protonated solvent signal.

Gel permeation chromatography (GPC) was performed on a Polymer Standard Service PSS (Mainz, Germany) SECurity system with the following specifications: pump mod. 1260 infinity, MDS RIdetector mod. 1260 infinity (Agilent Technologies, Santa Clara, California, USA), precolumn: $50 \times 8 \mathrm{~mm}$ PSS PFG linear M, and 2 columns: $300 \times 8 \mathrm{~mm}$ PSS PFG linear M (particle size $7 \mu \mathrm{m}$; pore size $0.1-1.000 \mathrm{~kg} / \mathrm{mol}$ ) with hexafluoroisopropanol (HFIP, containing $3 \mathrm{~g} /$ L potassium trifluoroacetate (KTFA)) as eluent calibrated against PEG standards with molar masses from 0.1 to $1000 \mathrm{~kg} / \mathrm{mol}$. The columns were held at $40{ }^{\circ} \mathrm{C}$ and the flow rate was set to $0.7 \mathrm{~mL} / \mathrm{min}$. Prior to each measurement, samples were dissolved in the eluent and filtered through $0.2 \mu \mathrm{m}$ PTFE filters (Rotilabo, Karlsruhe, Germany) to remove particles, if any.

Rolling ball viscosity experiments were performed on a LOVIS 2000 M microviscometer from Anton Paar (Graz, Austria) using a LOVIS 1.8 capillary and a steel ball of $1.5 \mathrm{~mm}$ diameter. Prior to viscosity measurements the density was determined at 5 and $40{ }^{\circ} \mathrm{C}$ using a DMA $4100 \mathrm{M}$ density meter from Anton Paar (Graz, Austria). A temperature scan from $5{ }^{\circ} \mathrm{C} \rightarrow 40{ }^{\circ} \mathrm{C}$ and $40{ }^{\circ} \mathrm{C} \rightarrow 5{ }^{\circ} \mathrm{C}$ of a 10,15 , and 20 wt $\%$ aqueous sample was performed in an automated angle mode with $3 \mathrm{~min}$ of temperature equilibration to establish the temperature-dependent dynamic viscosity.

For pyrene fluorescence assay, $40 \mu \mathrm{L}$ pyrene solution $(25 \mu \mathrm{M})$ in acetone was added to glass vials. The solvent was evaporated and $3 \mathrm{~mL}$ of polymer solutions of different concentrations in deionized water were added to yield final polymer concentrations of $1 \times 10^{-7} \rightarrow 5 \times$ $10^{-5} \mathrm{M}$ and a fixed pyrene concentration of $5 \times 10^{-7} \mathrm{M}$. The pyrene fluorescence emission spectra $\left(\lambda_{\text {exitation }}: 330 \mathrm{~nm}\right.$ at $25{ }^{\circ} \mathrm{C}$ ) were recorded on a FP-8300 spectrofluorometer system from Jasco (GrossUmstadt, Germany) between 360 and $400 \mathrm{~nm}$. To determine the critical micelle concentration (CMC), the $I_{1} / I_{3}$ ratios as a function of polymer concentration were fitted using the Boltzmann fit function, which is given by

$$
y=\frac{A_{1}-A_{2}}{1+e^{\left(x-x_{0}\right) / \Delta x}}+A_{2}
$$

where $A_{1}$ and $A_{2}$ are the upper and lower limits of the sigmoid, $x_{0}$ is the center of the sigmoid, and $\Delta x$ is directly related to the range where the abrupt change of the sigmoid occurs. The onset of an abrupt decrease is defined as the CMC and can be determined via eq 2, which is given by

$$
x(\mathrm{CAC})=x_{0}-2 \Delta x
$$

Rheology investigations were recorded on an Anton Paar (Ostfildern, Germany) Physica MCR 301 system utilizing a plateplate geometry ( $25 \mathrm{~mm}$ diameter) equipped with a solvent trap and Peltier element for temperature control. All aqueous samples were measured after complete dissolution in Millipore water and a concentration of 20 wt $\%$ at $5{ }^{\circ} \mathrm{C}$. A temperature sweep from 5 to 40 ${ }^{\circ} \mathrm{C}$ followed by a cooling sequence to $5{ }^{\circ} \mathrm{C}$ was carried out in an oscillation mode (angular frequency $10 \mathrm{rad} / \mathrm{s}$ and amplitude $0.1 \%$ ) and a constant heating or cooling rate of $0.1{ }^{\circ} \mathrm{C} / \mathrm{s}$. For investigations of viscoelastic behavior, the linear viscoelastic region (LVE) was determined by performing an amplitude sweep $(0.02 \% \rightarrow 500 \%)$ using a fixed angular frequency of $10 \mathrm{rad} / \mathrm{s}$. Subsequently, a frequency sweep $(0.1 \mathrm{rad} / \mathrm{s} \rightarrow 100 \mathrm{rad} / \mathrm{s})$ was performed at a fixed strain deformation of $0.1 \%$. For dispense plotting, rheological preconditions of the hydrogel are shear-thinning properties, defined force resistance profile, and fast structure recovery after deformation. For steady shear experiments, the control shear rate mode was used $(0.0011 / \mathrm{s} \rightarrow 1000$ $1 / \mathrm{s})$. The pronounced viscosity $\eta$ decrease was fitted using the power law expression (eq 3 )

$$
\eta=K \cdot(\dot{\gamma})^{n-1}
$$

where $K$ is the consistency index, $n$ is the flow index, and $j$ is the applied shear rate.

During a steady stress sweep $(5 \mathrm{~Pa} \rightarrow 1500 \mathrm{~Pa})$ the onset value of viscosity decrease is referred as the yield point of the hydrogel system. To investigate the structure recovery properties, two different recovery testing experiments were performed. During the ORO (oscillationrotational-oscillation) experiment, a low strain deformation of $0.5 \%$ is followed by a high shear rate of $100 \mathrm{1} / \mathrm{s}$. In the ROR (rotationaloscillation-rotational) experiment, a low shear rate region of $0.11 / \mathrm{s}$ is followed by a high strain of $100 \%$.

Dynamic light scattering (DLS) experiments were performed on an ALV SP125 (Langen, Germany) equipped with a He-Ne laser (22 mW, $\lambda=632.8 \mathrm{~nm}$ ) and a single optical avalanche photodiode detector or an ALV CGS-3 multidetection goniometry system (Langen, Germany) equipped with a He-Ne laser $(632.8 \mathrm{~nm})$ and eight optical avalanche photodiode detectors with a detector angle distance of $16^{\circ}$. Scattering angles from 30 to $125^{\circ}$ were measured with a $5^{\circ}$ angle interval for aqueous samples $\left(15,20,25\right.$, and $\left.40^{\circ}\right)$ and 30 to $70^{\circ}$ for a methanol sample $\left(20^{\circ} \mathrm{C}\right.$, correlation time $60 \mathrm{~s}$, average of $3-5$ runs). Prior to each measurement, samples were dissolved in methanol $(10 \mathrm{~g} / \mathrm{L})$ and filtered through $0.02 \mu \mathrm{m}$ Anotop membrane filters from Whatman GE Healthcare followed by Millex-LG $0.2 \mu \mathrm{m}$. After evaporation of methanol, the samples were dissolved in prefiltered (Millex-LG $0.2 \mu \mathrm{m}$ ) Millipore water (containing $2 \mathrm{mMNaNO}_{3}$ ) or methanol. Samples $(1 \mathrm{~g} /$ L) were filtered (Millex-LG $0.2 \mu \mathrm{m}$ ) into dust-free cuvettes under laminar flow. The decay of the electric field-time autocorrelation function (ACF) was fitted using biexponential fit functions (eq 4) with respect to polydispersities:

$$
g_{1}(t)=a_{1} \cdot e^{\left(-t / \tau_{1}\right)}+a_{2} \cdot e^{\left(-t / \tau_{2}\right)}
$$

with the amplitudes $a_{i}$ and the decay time $\tau_{i}=\frac{1}{q^{2} \cdot D_{i}}$, $q$ being the absolute value of the scattering vector and $D$ the translational diffusion coefficient which is indirectly proportional to the hydrodynamic radius $R_{\mathrm{h}}$ (Stokes-Einstein equation):

$$
R_{\mathrm{h}}=\frac{k_{\mathrm{B}} \cdot T}{6 \cdot \pi \cdot \eta \cdot D}
$$

with Boltzmann constant $k_{\mathrm{B}}$ and the viscosity of the solvent $\eta$.

For transmission electron microscopy (TEM) investigations, the polymer was dissolved in ultrapure water to a final concentration of 20 $\mathrm{g} / \mathrm{L}$. For the negative stain method, 400 mesh copper-rhodium grids (maxtaform) with a homemade carbon layer were glow-discharged in air for $1.5 \mathrm{~min}$ at medium power in a Harrick PDC-002 plasma cleaner. The $20 \mathrm{~g} / \mathrm{L}$ sample was diluted $(1 / 125)$ and $8 \mu \mathrm{L}$ was incubated on the grids for $1 \mathrm{~min}$ before blotting (Whatman filter paper No 50). Subsequently, the grids were washed with water $(3 x)$ and with $2 \%$ (w/ v) uranyl acetate $(3 \times)$. For imaging, a single-tilt room temperature holder in a FEI Tecnai T12 Spirit transmission electron microscope equipped with $\mathrm{a} \mathrm{LaB}_{6}$ emitter at $120 \mathrm{kV}$ was used. Images were recorded with an Eagle CCD camera under low-dose conditions. The micrographs were binned two times resulting in a pixel size of $4.4 \AA$ / pix at the specimen level.

The temperature-dependent ${ }^{1} \mathrm{H}$ NMR analysis was performed on a Bruker Avance III HD 600 spectrometer (Karlsruhe, Germany) operating at $600.4 \mathrm{MHz}$ with a BBFO $5 \mathrm{~mm}$ probe. ${ }^{1} \mathrm{H}$ NMR experiments at different temperatures $\left(5-40^{\circ} \mathrm{C}\right)$ of a $20 \mathrm{wt} \%$ hydrogel sample in $\mathrm{D}_{2} \mathrm{O} / \mathrm{H}_{2} \mathrm{O}(1: 1)$ were performed without sample spinning and 16 scans. The sample was kept for $10 \mathrm{~min}$ at the desired temperature prior to each measurement. Temperature calibration was 
done using 4\% MeOH in MeOD and 80\% ethylene glycol in DMSO- $\mathrm{d}_{6}$. All recorded spectra were referenced using the temperature-dependent HDO signal. For a more quantitative characterization of the temperature-induced phase transition, the fraction $p$ was calculated with the integrals $I(T)$ and $I\left(T_{0}\right)$ at the respective temperatures $T$ and $T_{0}$ using the following equation: ${ }^{44}$

$$
p=1-\frac{I(T)}{I\left(T_{0}\right) * \frac{T_{0}}{T}}
$$

The highest signal intensity was measured at $40{ }^{\circ} \mathrm{C}$, which was chosen to be $T_{0}$. Reductions of signal intensity caused by inhibited molecular mobility due to gelation and viscosity increase were quantified by values of $p<0$.

For small-angle X-ray scattering (SAXS) experiments, an in-house setup at the Chair of X-ray Microscopy at the University of Würzburg was used, which was built by Fraunhofer EZRT (Fürth, Germany). It consists of a MicroMax-007 HF X-ray source (Rigaku, Japan) and an EIGER R 1M detector unit (Dectris, Switzerland). The sampledetector distance can be varied between $5 \mathrm{~cm}$ and $3.5 \mathrm{~m}$, which corresponds to possible $Q$-values between 0.005 and $5 \AA^{-1}$. The complete setup is operated in a vacuum below $0.1 \mathrm{mbar}$ to reduce air scattering. The sample solution $(20 \mathrm{wt} \%)$ was placed in quartz capillaries (inner diameter: $1 \mathrm{~mm}$, wall thickness: $10 \mu \mathrm{m}$ ) (Hampton Research, Aliso Viejo, California), which were positioned perpendicularly to the X-ray beam. The presented experiments were done at sample-detector distances of 57,565 , and $1560 \mathrm{~mm}$ with an integration time of $15 \mathrm{~min}$ for the shortest distance and $240 \mathrm{~min}$ for the two longer configurations. All distances were calibrated using a silver behenate standard sample. Data were acquired for different temperatures between 5 and $40{ }^{\circ} \mathrm{C}$. To achieve thermal equilibrium, the sample was kept at the desired temperature for $15 \mathrm{~min}$ prior to each measurement. The SAXS data, which were obtained at the two largest distances, were calibrated in terms of absolute intensities using glassy carbon as a secondary calibration standard. ${ }^{45,46}$ The scattering curves of the hydrogel were obtained by azimuthal integration and corrections taking the sample thickness, X-ray transmission, detector accuracy, setup geometry, and solvent scattering into account, by following the standard procedures described in the literature. ${ }^{47}$ A detailed description of the fitting procedure using a hard sphere model can be found in the Supporting Information.

For scanning electron microscopy (SEM) of the native hydrogel structure, we investigated a cryogenic sample preparation procedure. For this, hydrogel samples were placed between two aluminum holders $(d=3 \mathrm{~mm})$, both containing a notch with a diameter of $2 \mathrm{~mm}$, enclosing the sample and rapidly frozen in slush nitrogen $(\mathrm{SN})$ at $-210^{\circ} \mathrm{C}$. The samples were then transferred into a sputter coater with a Leica EM VCT100 cryo-shuttle at $-140{ }^{\circ} \mathrm{C}$ (Leica Microsystems ACE 400, Wetzlar, Germany). Here, the upper half of the sample was knocked off to create a fresh fractured surface and freeze-etched at $-85{ }^{\circ} \mathrm{C}$ for 15 min under vacuum $\left(<1 \times 10^{-3} \mathrm{mbar}\right)$. The samples were finally sputtered with $3 \mathrm{~nm}$ platinum and transferred with the cryo-shuttle into the SEM chamber. The morphology of the fractured surfaces was imaged at $-140^{\circ} \mathrm{C}$, by detecting SE using acceleration voltages of 2 or 8 $\mathrm{kV}$.

To investigate the printability of 20 wt \% hydrogel samples, a compact bench-top 3D bioprinter (BioX, Cellink, Sweden) working on the principle of an extrusion-based technology was used. The printhead and the print bed were cooled to $8{ }^{\circ} \mathrm{C}$ prior to each fabrication process. The printing speed was set to $10 \mathrm{~mm} / \mathrm{s}$ and a pressure of $120-160 \mathrm{kPa}$ was applied (nozzle: $25 \mathrm{G}$, stainless steel, length of $6.35 \mathrm{~mm}$ ). First, the printing resolution was investigated by increasing the strand-center to strand-center distance stepwise from $0.5 \mathrm{~mm} \rightarrow 0.75 \mathrm{~mm} \rightarrow 1 \mathrm{~mm} \rightarrow$ $1.5 \mathrm{~mm} \rightarrow 2 \mathrm{~mm}$ (layer height $0.25 \mathrm{~mm}$ ). To investigate the steadiness of the hydrogel, a strand collapse test was investigated as described by Ribeiro et al. ${ }^{48}$ The distance between the two edition points increased from $0.1 \rightarrow 0.2 \rightarrow 0.4 \rightarrow 0.9 \rightarrow 1.7 \mathrm{~cm}$. Finally, real 3D-printing was performed by printing a 20-layered tubular construct of $5 \mathrm{~mm}$ total height and $5 \mathrm{~mm}$ diameter using the parameters already described.
To evaluate the cytocompatibility of POx/alginate (20:1 wt \%) bioinks, the viability of embedded NIH 3T3 cells (ATCC, Germany, 1 $\mathrm{Mio} / \mathrm{mL}$ ) was investigated. As a reference, sodium alginate (VIVA Pharm, PH176, $1 \mathrm{wt} \%$ ) was used. After homogenous cell distribution at $37^{\circ} \mathrm{C}$ a simple one-layered square structure (Figure S6) was printed at $10^{\circ} \mathrm{C}$ with a printing pressure of $50 \mathrm{kPa}$ and a printing speed of $5 \mathrm{~mm} / \mathrm{s}$. Stabilization after printing was performed using a $0.1 \mathrm{M} \mathrm{CaCl}_{2}$ aqueous solution $(10 \mathrm{~min})$. The cross-linked scaffolds were incubated for $24 \mathrm{~h}$ at $37{ }^{\circ} \mathrm{C}$ in cell culture medium (DMEM high glucose (Gibco from Sigma-Aldrich)) supplemented with $1 \%$ pen/strep, $2 \%$ glutamine (Thermo Fisher), and 10\% bovine calf serum from Corning under controlled conditions ( $5 \% \mathrm{CO}_{2}, 95 \%$ relative humidity). To assess the cell viability, printed NIH $3 \mathrm{~T} 3$ cells were stained inside the scaffolds using Calcein AM (Invitrogen, Thermo Fisher) after $24 \mathrm{~h}$ of cultivation, whereas blue nuclei acid stain (DAPI) (Thermo Fisher) was used to visualize nuclei of all embedded cells. ImageJ software was used to determine the cell viability using automatic cell counting in three fluorescence images $(n=3)$ of three different samples $(n=3)$ obtained with an epifluorescence microscope (Zeiss Observer, Germany) using the following equation:

$$
\text { cell viability }=\frac{[\text { number of living cells }]}{\text { [number of cell nuclei }]}
$$

\section{RESULTS AND DISCUSSION}

Two batches of an ABA-triblock copolymer $\mathrm{PMeOx}_{35}-b$ $\mathrm{PPhenEtOx}_{15}-b$-PMeOx ${ }_{35}$ (=A-PPhenEtOx-A) (Scheme 1B) were synthesized by living cationic ring-opening polymerization (LCROP) and characterized by ${ }^{1} \mathrm{H}$ NMR spectroscopy (Figures S2 and S5) and GPC (Figures S1 and S4). The successful termination using different terminating agents (P1: 1-Bocpiperazine (BOC-Pip), P2: ethyl isonipecotate (EIP)), the comparable degree of polymerization of approx 90 repeating units, and a ratio of $\mathrm{PMeOx} / \mathrm{PPhenEtOx}$ of 4 were verified by the corresponding signal intensities in ${ }^{1} \mathrm{H}$ NMR spectra.

Via GPC, the number average molar mass $M_{\mathrm{n}}$ and the dispersity $Ð\left(M_{\mathrm{w}} / M_{\mathrm{n}}\right)$ of both batches were compared after completion of every single block and purification of the final polymer product. Both batches show reasonably narrow molar mass size distributions $(Ð(\mathrm{P} 1)=1.12, Ð(\mathrm{P} 2)=1.17)$ and an increase in the number average molar mass during the polymerization reaction, indicative of the living polymerization. The primary structural difference between these novel polymers in comparison to the previously described aromatic amphiphiles is one additional methylene group in the sidechains of every repeat unit in the central B block, which leads to significantly altered physicochemical properties. Very similar to previously described ABA-type amphiphiles, the novel polymers form stable nanoscale micelles in aqueous solutions above the CMC. $^{42,49}, 50$ The investigated A-PPhenEtOx-A polymer exhibited a rather low CMC of $0.3 \mu \mathrm{M}$ as determined by pyrene fluorescence measurement in dependency of the polymer concentration (Figure 1A,B). The hydrodynamic radius of these nanoscale aggregates was determined via DLS at different scattering angles (Figure 1C,D). In the nonselective solvent, methanol, the hydrodynamic radius of an individual polymer chain was determined as ca. $2.4 \mathrm{~nm}$. In contrast, in the selective solvent water, the polymers self-assemble into micelles with a hydrodynamic radius of ca. $8 \mathrm{~nm}$. The low dependence on the scattering angle $\left(30 \rightarrow 125^{\circ}\right)$ suggests spherical micelles of low polydispersity (Figure 1D). The spherical morphology of the micelles could be further corroborated by means of TEM (Figure 1E).

Inspired by our previous finding for a similar $A B A$ triblock, ${ }^{39,40}$ we investigated the thermoresponsive properties. 
A)

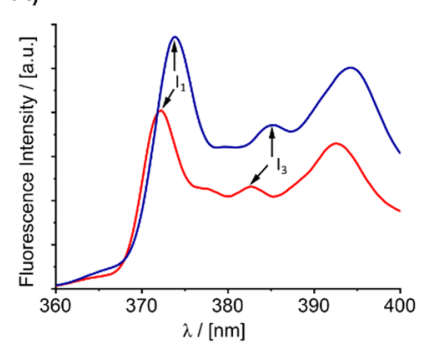

B)

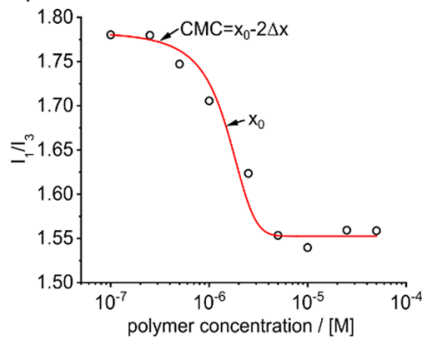

C)

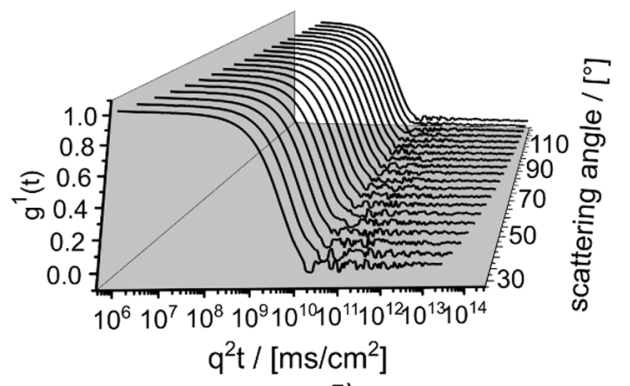

D)

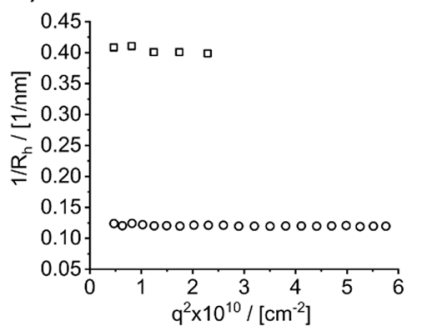

E)

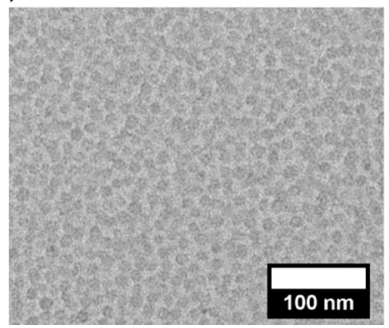

Figure 1. Self-assembly of A-PPhenEtOx-A at low polymer concentration in aqueous solution. (A) Characteristic pyrene emission spectra at $25^{\circ} \mathrm{C}$ and polymer concentrations of $10^{-7} \mathrm{M}$ (red) and $10^{-5}$ $\mathrm{M}$ (blue). (B) $I_{1} / I_{3}$ intensity ratio of the pyrene signal at different polymer concentrations fitted with the Boltzmann fit function $\left(R^{2}\right.$ : 0.99707). (C) Autocorrelation functions of DLS experiments of $1 \mathrm{~g} / \mathrm{L}$ aqueous polymer solution containing $2 \mathrm{mM} \mathrm{NaNO}_{3}$ at $20{ }^{\circ} \mathrm{C}$ and different scattering angles $\left(30 \rightarrow 125^{\circ}\right.$ in $5^{\circ}$ steps $)$. (D) Inverse hydrodynamic radius in dependency of the scattering vector $q^{2}$ of $1 \mathrm{~g} / \mathrm{L}$ aqueous polymer solution containing $2 \mathrm{mM} \mathrm{NaNO}_{3}$ (circle) compared to $1 \mathrm{~g} / \mathrm{L}$ polymer solutions in methanol as nonselective solvent (square). (E) TEM image from aqueous polymer solution stained with uranyl acetate showing spherical micelles.

Indeed it was observed that when the concentration exceeded 20 wt \%, a hydrogel was formed at low temperature (Figure 2A,B (P2), Figure S3 (P1)). However, investigating this phenomenon in more detail, crucial differences in gel strength, critical concentration, and temperature compared to the previously described material $\left(\mathrm{PMeO}_{35}-b\right.$-PPheOzi $\left.{ }_{15}-b-\mathrm{PMeOx}_{35}\right)$ were found. The thermoresponsive behavior was evaluated by rolling ball viscosimetry at different concentrations $(10,15$, and $20 \mathrm{wt}$ $\%)$ combined with a heating $\left(5^{\circ} \mathrm{C} \rightarrow 40{ }^{\circ} \mathrm{C}\right)$ and cooling $\left(40^{\circ} \mathrm{C}\right.$ $\rightarrow 5{ }^{\circ} \mathrm{C}$ ) ramp. At 10 and 15 wt $\%$, the viscosity decreases steadily with increasing temperature $\left(\eta_{5}{ }^{\circ} \mathrm{C}(10 \mathrm{wt} \%)=9 \mathrm{mPas}\right.$ $\rightarrow \eta_{40}{ }^{\circ} \mathrm{C}(10$ wt $\%)=3 \mathrm{mPas}, \eta_{5}{ }^{\circ} \mathrm{C}(15 \mathrm{wt} \%)=50 \mathrm{mPas} \rightarrow$ $\left.\eta_{40}{ }^{\circ} \mathrm{C}(10 \mathrm{wt} \%)=11 \mathrm{mPas}\right)$ without any notable features. The cooling and heating ramps yield very similar values. In contrast, at 20 wt \% the viscosity increases very fast below a critical temperature of approx. $22{ }^{\circ} \mathrm{C}$ eventually trapping the ball, whereupon no further values are obtained (Figure 2C). Increasing the temperature, the polymer solution again flows freely forming a moderately viscous solution with dynamic viscosity values of approx. $70 \mathrm{mPas}$ at $40^{\circ} \mathrm{C}$. A notable hysteresis
A)

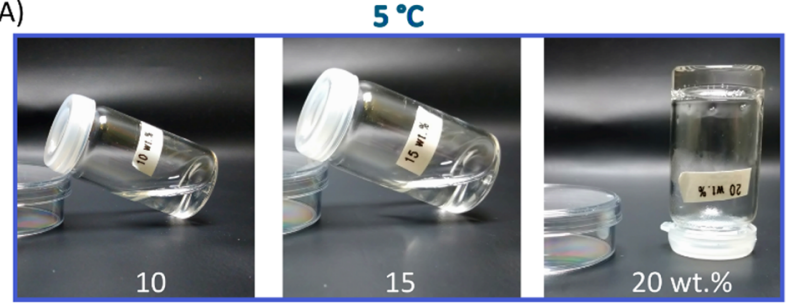

B)
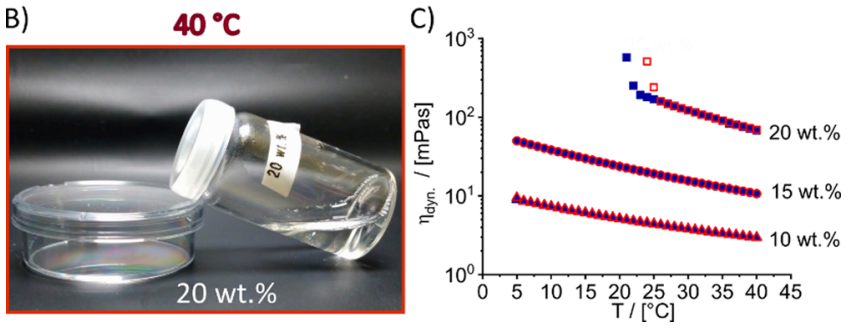

Figure 2. Increasing concentration of A-PPhenEtOx-A leads to hydrogel formation. (A) Pictures of aqueous solutions at $5{ }^{\circ} \mathrm{C}$ and different polymer concentrations $(10 \rightarrow 15 \rightarrow 20$ wt \%). (B) Photographic images of aqueous solution at $40{ }^{\circ} \mathrm{C}$ and a polymer concentration of 20 wt \%. (C) Temperature-dependent viscosity measurements (rolling ball system) at different polymer concentrations (blue and filled symbols: cooling, red and open symbols: heating).

of approximately $3{ }^{\circ} \mathrm{C}$ was observed in this experiment. In addition, we characterized the inverse gelation by rheology (Figure S7). Again, a hysteresis of the gel temperature $T_{\text {gel }}\left(\mathrm{G}^{\prime}=\right.$ $\left.\mathrm{G}^{\prime \prime}\right)$ between heating $\left(T_{\text {gel }}=25^{\circ} \mathrm{C}\right)$ and cooling $\left(T_{\text {gel }}=22^{\circ} \mathrm{C}\right)$ can be seen. The gelation is fully reversible and a stable hydrogel $\left(G^{\prime}>>G^{\prime \prime}\right)$ below $T_{\text {gel }}$ was observed.

Compared to the recently described ABA triblocks featuring a PPheOzi hydrophobic B block, there are notable differences in the present system. First, a significantly higher concentration is needed $\left(c_{\text {gel }}[\right.$ A-PPheOzi-A $]=5$ wt $\%^{39}$ vs $c_{\text {gel }}[$ A-PPhenEtOx-A $]$ $=20$ wt $\%)$. Second, the critical temperature is lower $\left(T_{\text {gel }}[\mathrm{A}\right.$ PPheOzi-A $]=32{ }^{\circ} \mathrm{C}^{39}$ vs $T_{\text {gel }}[\mathrm{A}-\mathrm{PPhenEtOx}-\mathrm{A}]=22-25^{\circ} \mathrm{C}$ ). Third, the gelation kinetics is much faster compared to the previous system (slow gelation ${ }^{39}<->$ rapid gelation).

To investigate the thermoresponsive aggregation on a molecular level, temperature-dependent ${ }^{1} \mathrm{H}$ NMR experiments were conducted (Figure $3 \mathrm{~A}, \mathrm{~B})$. At $5{ }^{\circ} \mathrm{C}$, the signals attributed to the polymer backbone $(3.45 \mathrm{ppm})$ and $\mathrm{CH}_{3}$-sidechain $(2.00$ $\mathrm{ppm}$ ) of the hydrophilic building blocks are visible, albeit broad and featureless. In the aromatic region $(6.92 \mathrm{ppm})$ of the spectrum, no signals are visible at $5{ }^{\circ} \mathrm{C}$. This can be readily explained by very short transversal relaxation times $\mathrm{T}_{2}{ }^{*}$ of the moieties in the hydrophobic micellar core, which are densely packed and apparently less hydrated so that efficient spin-spin relaxation can occur. As previously discussed, a gradual increase of the temperature from 5 to $40{ }^{\circ} \mathrm{C}$ causes liquefaction, and the signals of the hydrophilic as well as the hydrophobic part become narrower and more defined (Figure 3A,B) as molecular mobility increases. Most interestingly, this is particularly visible in the aromatic region of the spectrum. An increasing, albeit still featureless signal is visible with increasing temperature, which is caused by more flexible units of the hydrophobic core. Likewise, the reversibility of aggregation could be demonstrated by a subsequent cooling sequence, whereby the initial aggregation is achieved (Figure 3B). A more quantitative insight was obtained by comparing the integrated NMR intensities of different polymer signals as a function of temperature (Figure 3C). A $p$ value (eq 6 ) of zero indicates the highest mobility in the liquid 

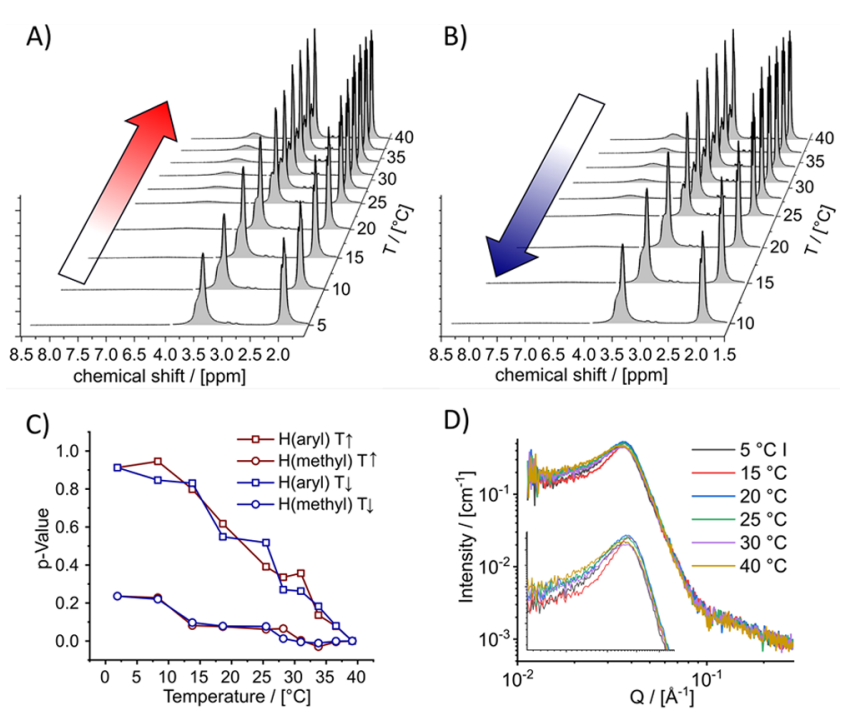

D)

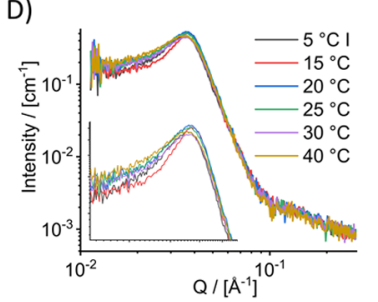

E)

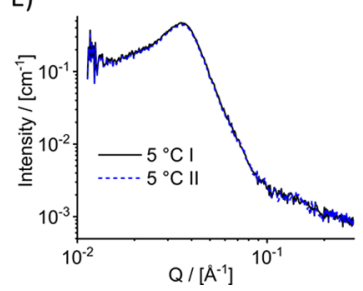

$\mathrm{A}^{39}$ showed a constant $p$-value in the gel state and a rapid $p$-value decrease upon liquefaction. The hydrophilic shell and the backbone are affected to a much lesser extent, similar to previous reports on the aggregation behavior of thermoresponsive phase separation of different POx-based polymers. ${ }^{44}$ In contrast to DLS, the polymer sol/gel could be investigated at concentrations needed for hydrogel formation (20 wt \%) using SAXS. The scattering curves suggest a feature size of $16-18 \mathrm{~nm}$ with only small changes with changing the temperature (Figure 3D). However, the small changes observed proved to be fully reversible as evidenced by scattering curves at $5{ }^{\circ} \mathrm{C}$ being superimposable after a heating/cooling cycle to $40{ }^{\circ} \mathrm{C}$ (Figure $3 \mathrm{E}$ ). To characterize the scattering curves in more detail, hardsphere model fit functions were employed (detailed information in the Supporting Information and Table S1). In particular, we focused to obtain the correlation length $\xi$, the distance in which neighboring micelles correlate with each other. Interestingly, $\xi$ increased with decreasing temperature with a strong increase in particular between 15 and $5{ }^{\circ} \mathrm{C}$. An increased correlation length means that the micelles interact over a greater distance. Interestingly, on comparing the temperature-dependent rheology and correlation length it, becomes apparent that the correlation is not completely straightforward.

In fact, the correlation length only increases strongly below the $T_{\text {gel }}$, at $15{ }^{\circ} \mathrm{C}$ and below, while gelation $\left(G^{\prime} \geq G^{\prime \prime}\right)$ occurs already at $22{ }^{\circ} \mathrm{C}$. Interestingly, below $15{ }^{\circ} \mathrm{C}$, the p-value for the pMeOx shows a marked increase, indicating reduced mobility. Looking more closely at the temperature-dependent rheology, the longer correlation lengths coincide with the formation of a plateau for both $G^{\prime}$ and $G^{\prime \prime}$. In contrast, the values for $G^{\prime}$ and $G^{\prime \prime}$ at $T_{\text {gel }}$ are about three orders of magnitudes lower than at this plateau. It appears at this point that the hydrogel formation of APPhenEtOx-A is caused by densely packed spherical micelles with reduced mobility at low temperature due to increased intermicelle correlation, somewhat resembling the aggregation behavior of Pluronic F127. However, Pluronic F127 forms a hydrogel upon heating because of a thermoresponsive poly(propylene glycol) block whereas A-PPhenEtOx-A does so upon cooling and does not contain a polymer block which is thermoresponsive in itself. Accordingly, it appears obvious that on the molecular level, the thermogelation of APPhenEtOx-A and Pluronic F127 is not related. Also, comparing to the wormlike hydrogel system comprising A-PPheOzi-A ${ }^{40}$

state $\left(40{ }^{\circ} \mathrm{C}\right)$. The aromatic $\mathrm{CH}$-protons are most strongly influenced yielding high $p$-values (close to $1=$ little signal intensity left) in the gel state. Liquification due to increased temperature caused an increasing signal resulting in a steady $p$ value decrease up to $40{ }^{\circ} \mathrm{C}$ for the hydrophobic signals. The previously described slow gelling system based on A-PPheOzi-

Scheme 2. Self-Assembly of the ABA-Type Triblock Copolymer and Inverse Thermogelation ${ }^{a}$

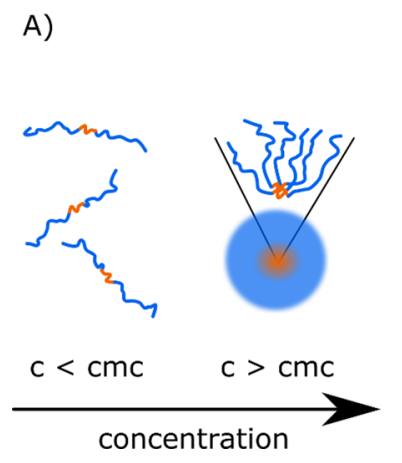

B)

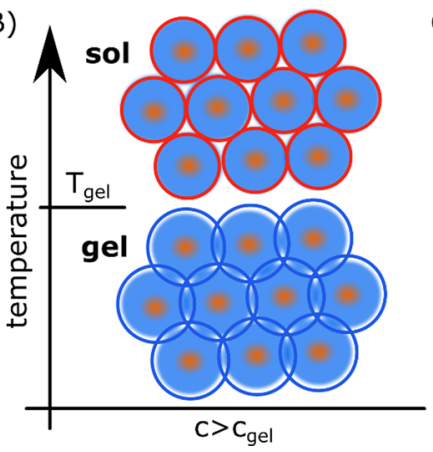

C) increased correlation
upon cooling

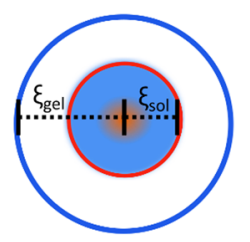

a (A) At very low polymer concentration single polymer chains are present. The aqueous polymer solution has a low viscosity. Above the CMC, the amphiphile self-assembles into spherical micelles. (B) Increase in concentration above $T_{\text {gel }}$ leads to a micellar solution with moderate viscosity and macromolecular mobility. Decrease in temperature below $T_{\text {gel }}$ leads to an increase in the intermicelle correlation, physical network formation, and a macroscopic gelation. (C) Schematic illustration of the different correlation lengths at different temperatures. Upon cooling, the correlation of individual micelles increases. 
A)

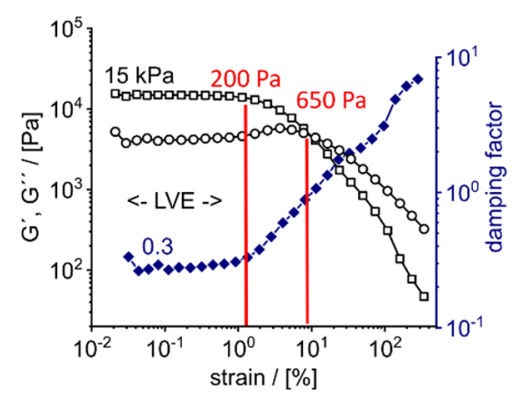

D)

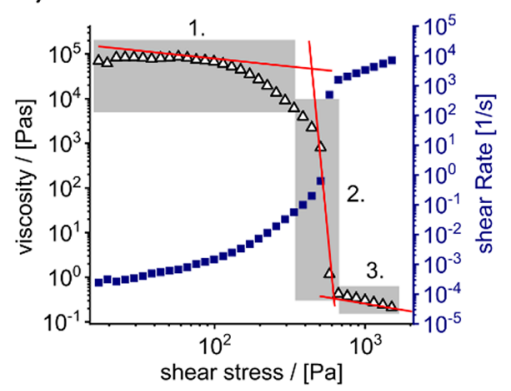

G)

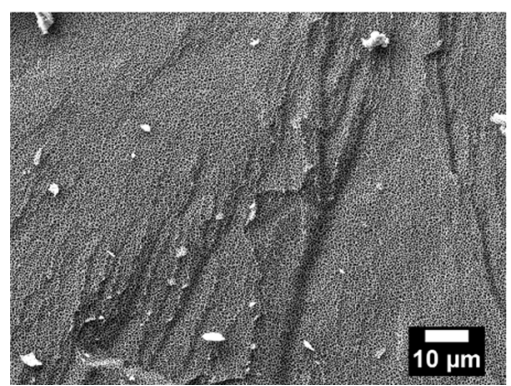

B)

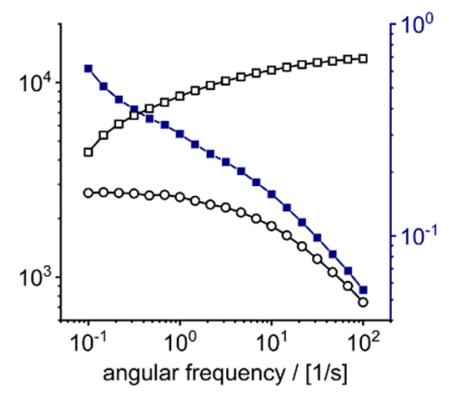

E)

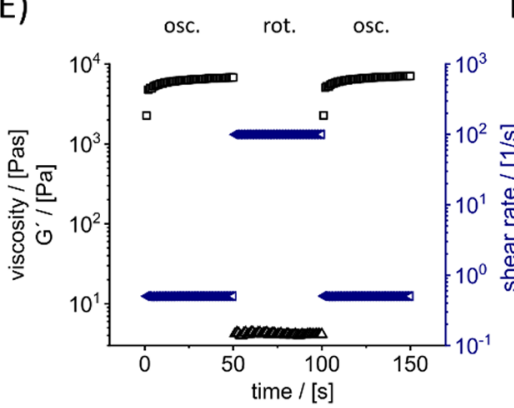

F)
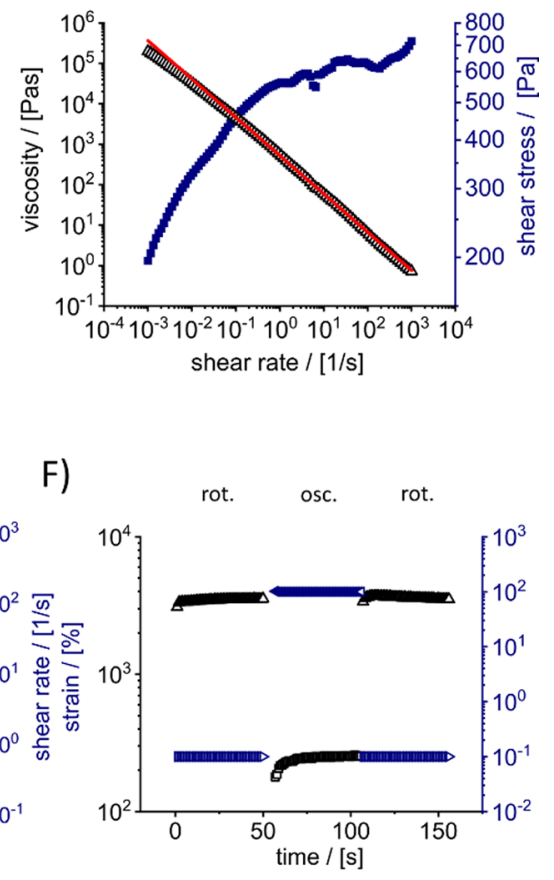

H)

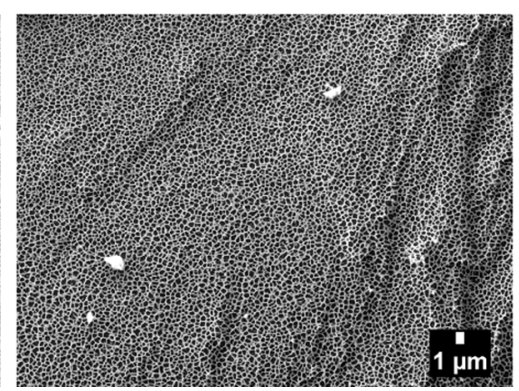

I)

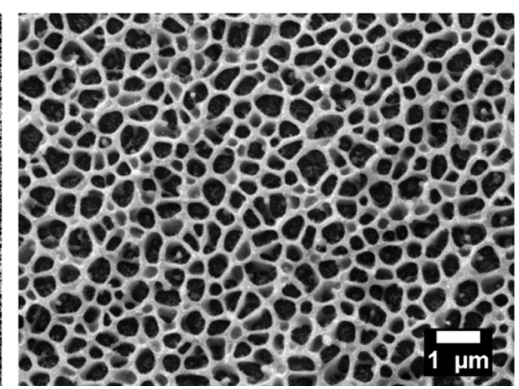

Figure 4. Rheological characterization for dispense plotting applications and cryoSEM analysis of the A-PPhenEtOx-A hydrogel at $5^{\circ} \mathrm{C}$. (A) Amplitude sweep of the $20 \mathrm{wt} \%$ hydrogel at $5^{\circ} \mathrm{C}$ and an angular frequency of $10 \mathrm{rad} / \mathrm{s}$ with storage moduli $\left(\mathrm{G}^{\prime}=\right.$ square $)$, loss moduli $\left(\mathrm{G}^{\prime \prime}=\right.$ circle $)$, and damping factor (filled square) is shown. (B) Frequency sweep with a fixed amplitude of $0.1 \%$. (C) Viscosity (triangle) and shear stress (filled square) in dependency of the applied shear rate for a $20 \mathrm{wt} \%$ hydrogel. (D) Viscosity (triangle) and shear rate (filled square) as a function of the applied shear stress in the steady shear stress experiment. (E) ORO recovery test of the hydrogel. (F) ROR three-step recovery. (G-I) CryoSEM images of the 20 wt $\%$ hydrogel at $1 \mathrm{k} \times, 2 \mathrm{k} \times$ and $10 \mathrm{k} \times$ magnification.

with different slope regions in the SAXS pattern, significant differences were obtained. To summarize, the amphiphile selfassembles into spherical micelles $\left(R_{\mathrm{h}}\left(15^{\circ} \mathrm{C}\right)=8.5 \pm 0.1 \mathrm{~nm}, R_{\mathrm{h}}\right.$ $\left(25^{\circ} \mathrm{C}\right)=8.4 \pm 0.1 \mathrm{~nm}$, and $\left.R_{\mathrm{h}}\left(40^{\circ} \mathrm{C}\right)=8.5 \pm 0.2 \mathrm{~nm}\right)$ at all investigated temperatures above the CMC $(0.3 \mu \mathrm{M})$. In comparison, the previously described inverse gelling system with PheOzi instead of PhenEtOx as hydrophobic repeat units showed a pronounced temperature dependency of the apparent $R_{\mathrm{h}}\left(R_{\mathrm{h}}\left(15^{\circ} \mathrm{C}\right)>100 \mathrm{~nm}\right)$ connected to a change in micellar morphology from spherical to worm. ${ }^{40}$ Here, the increased concentration leads to the formation of highly concentrated dispersion of spherical micelles, similar to the situation well known for Pluronic F127. However, in contrast to the present system, Pluronic F127 only forms micelles above the critical temperature of the poly(propylene glycol) blocks. Decreasing the temperature resulted in reduced micellar mobility which was observed due to an increasing correlation length between neighboring micelles, consequently leading to the inverse gelation (Scheme 2).

As previously mentioned, stimuli-responsive hydrogels have been utilized in different biomaterial applications. ${ }^{51}$ Recently, different approaches were established to overcome limitations in the so-called biofabrication window, especially in bioprinting. ${ }^{52}$ One approach is to improve printability by support materials and sacrificial materials, while retaining cell viability by utilizing biological components, cell-friendly cross-linking, and mild printing conditions. The search for suitable hydrogel platforms is an ongoing challenge due to multiple specific and sometimes contradictive requirements. Appropriate gelation kinetics, good printability, and cytocompatibility are key requirements for hydrogels during bioink design. Therefore, the viscoelastic properties of the present inverse thermogelling platform were thoroughly investigated via rheology. In the pronounced viscoelastic region (LVE, end of LVE and flow point $\left(G^{\prime}=\right.$ 


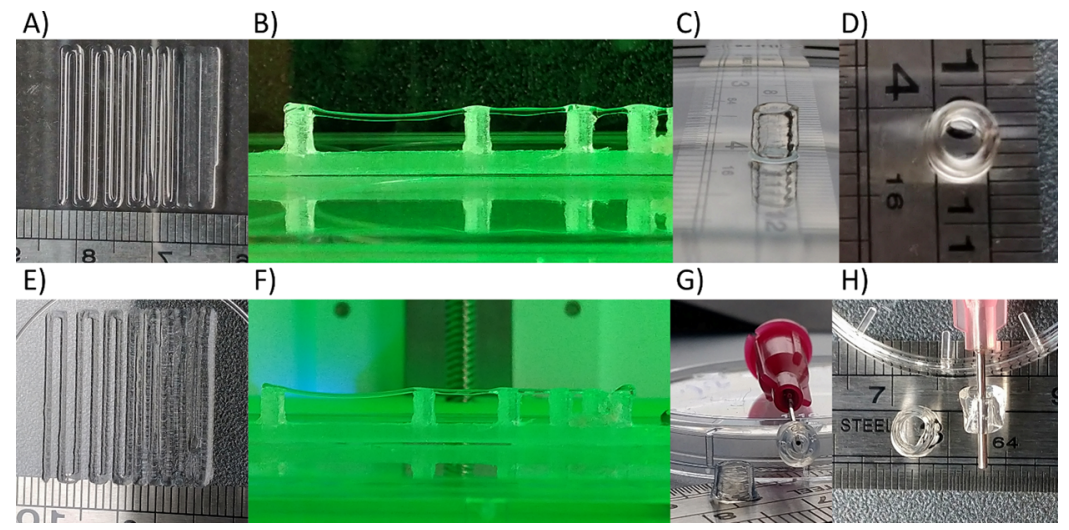

Figure 5. Extrusion printing of a A-PPhenEtOx-A 20 wt \% hydrogel (A-D) and a hydrogel blend of A-PPhenEtOx-A and alginate (E-H) at $8{ }^{\circ} \mathrm{C}$ (nozzle $25 \mathrm{G}$, speed: $10 \mathrm{~mm} / \mathrm{s}$ ). (A, E) Filament fusion test to visualize printing resolution. (B, F) Filament collapse test by printing bridges of increasing distances. $(\mathrm{C}, \mathrm{D}, \mathrm{G}, \mathrm{H})$ Printed 20 layer $(5 \times 5 \mathrm{~mm}$ cylindrical tube structures) constructs (top and side view).
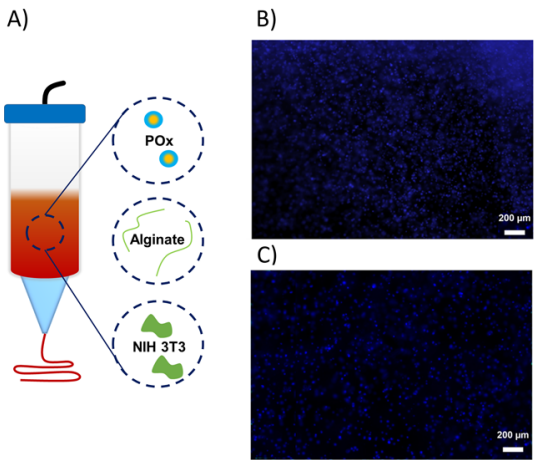

POx/Alginate

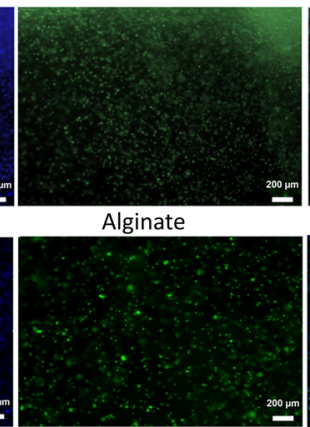

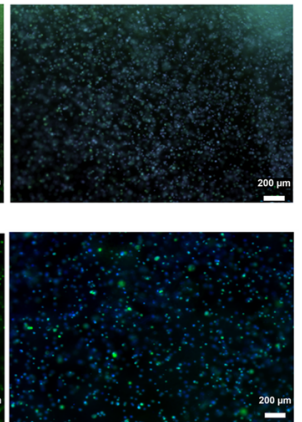

D)

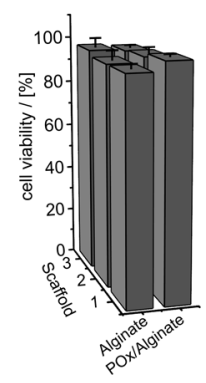

Figure 6. Cytocompatible bioprinting of POx/alginate-based bioinks using NIH 3 T3 cells (A). (B, C) Single and merged fluorescence images of embedded NIH 3 T3 cells in POx/alginate (upper row) and alginate (bottom row) stained with DAPI (blue) and Calcein AM (green) after printing, stabilization with $\mathrm{CaCl}_{2}$, and $24 \mathrm{~h}$ of incubation. (D) Cell viability of $\mathrm{NIH} 3 \mathrm{~T} 3$ cells in three different printed scaffolds of alginate and POx/alginate hydrogel blend.

$\mathrm{G}^{\prime \prime}$ ) marked with red lines), a constant storage modulus $\mathrm{G}^{\prime}$ of $14.8 \pm 0.3 \mathrm{kPa}$ was obtained with a low damping factor of $0.3 \pm$ 0.02 , which suggest a stable viscoelastic hydrogel (Figure 4A). Interestingly, the hydrogel exhibits a profound dependency on the frequency (Figure 4B). Increasing the frequency leads to increased $G^{\prime}$ and decreased $G^{\prime \prime}$ values, i.e., the solid-like character is amplified with the dampening factor reaching values $<0.03$ at $500 \mathrm{~s}^{-1}$. To utilize a hydrogel as 3D-printable ink in different printing applications, three major rheological considerations must be addressed, namely, pronounced shear-thinning, defined force resistance, and fast structure recovery properties. ${ }^{53}$ With the increasing shear rate, the viscosity of the present hydrogel decreases following a power law expression with a flow index of $0.05 \pm 0.004$, indicating very pronounced shearthinning (Figure 4C). Even at very low shear rates of $0.001 \mathrm{~s}^{-1}$, no viscosity plateau is reached and shear stress values of ca. 200 $\mathrm{Pa}$ are measured at this point. In contrast, at very high shear rates of $1000 \mathrm{~s}^{-1}$, the viscosity values are very low (ca. $1 \mathrm{~Pa} \mathrm{~s}$ ) with shear stress values increasing up to $750 \mathrm{~Pa}$. To obtain the yield point and the force resistance of the hydrogel, the viscosity was plotted as a function of the applied shear stress and split up in three regions (Figure 4D). Below the critical stress, almost constant viscosity values between $10^{4}$ and $10^{5}$ Pas with very low shear rates (between $10^{-4}$ and $10^{-5} 1 / \mathrm{s}$ ) were determined (1). At a certain stress, the viscosity decreases very fast with increasing shear rate values to low viscosity values (2). The onset of $440 \mathrm{~Pa}$ is determined via two tangents. At high shear stress values of $800 \rightarrow 1500 \mathrm{~Pa}$, plateau values for the viscosity and shear rate of 0.1 Pas and $10^{3} \rightarrow 10^{4} \mathrm{~s}^{-1}$ are reached (3). Structure recovery was tested using a three-step test, designed as ORO and ROR experiments, altering low and high strain regimes (Figure $4 \mathrm{E}, \mathrm{F})$. In both cases, the structure recovered very fast and the initial values were obtained during the experiments, which we interpret to show rapid and complete structure recovery. To obtain insights into the hydrogel morphology, we conducted cryogenic scanning electron microscopy (cryoSEM) (Figure $4 \mathrm{G}-\mathrm{I}$ ). A rather homogenous porous structure with pores in the range of several hundred nanometers was obtained. In comparison to a recently described hydrogel based on a APPheOzi-A triblock copolymer, the presently described APPhenEtOx-A hydrogel has significantly smaller pores. ${ }^{39}$ The strong frequency dependence in combination with smaller pore sizes compared to the previously published hydrogels consisting of wormlike micelles further underlines the differences between the individual gelation mechanism. In summary, the novel hydrogel shows promising viscoelastic characteristics for bioprinting.

Based on favorable rheological properties, the printability of the hydrogel was investigated using extrusion-based printing (Figure 5A-D). Additionally, a hydrogel blend comprising the POx-based hydrogel and alginate was prepared, printed, and stabilized with $\mathrm{CaCl}_{2}$ (Figure 5E-H). Alginate hydrogels are widely used in biofabrication as alginate is easily cross-linked by the addition of $\mathrm{CaCl}_{2}$ after printing to stabilize the constructs. 
However, the printability of pure sodium alginate without the addition of viscosity enhancers or modifications is very low. ${ }^{54}$ First, we screened for the highest achievable resolution by printing hydrogel lines with a decreasing line distance, from $2 \rightarrow$ $1.5 \rightarrow 1 \rightarrow 0.75 \rightarrow 0.5 \mathrm{~mm}$ (Figure 5A,E). Even at a layer distance of $0.75 \mathrm{~mm}$ every individual strand could be distinguished and no strand fusion was observed. Additionally, the collapse of suspended filaments was investigated. ${ }^{48}$ A free hanging hydrogel strut over a total distance of $1.7 \mathrm{~cm}$ could be realized without significant strand sag (Figure 5B,F). Based on these promising results, a first real $3 \mathrm{D}$ construct was printed (Figure 5C,D,G,H, Supporting Movie 1). A 20-layered cylindrical construct with a diameter of $5 \mathrm{~mm}$ and a total height of $5 \mathrm{~mm}$ was printed. The structure stayed intact, and no significant collapse was observed. The structure of the stabilized $\mathrm{POx} /$ alginate tubular construct was preserved even after $24 \mathrm{~h}$ of incubation in PBS solution at $37^{\circ} \mathrm{C}$ (Figure 5G,H).

Finally, a first bioprinting process was performed and the cytocompatibility was investigated using the POx/alginate (20:1 wt \%) hydrogel blend in comparison to pure alginate (1 wt \%) (Figure 6A-D) after $24 \mathrm{~h}$. After dissolving the polymers in cell culture medium, the cells were dispersed in the homogeneous free flowing viscous liquid at $37^{\circ} \mathrm{C}$. The relatively high viscosity of the bioink precursor at $37{ }^{\circ} \mathrm{C}$ (see Figure 2) prevented significant cell sedimentation. The bioink blend was transferred to the precooled bioprinter equipped with a cooling system. Using the pronounced inverse gelation of A-PPhenEtOx-A as described above, cell-laden constructs were obtained and stabilized via cross-linking of alginate using $\mathrm{CaCl}_{2}$ aqueous solution. After $24 \mathrm{~h}$ of incubation, cell staining was performed and quantified. Overall, the bioprinting process of POx-based bioinks was found to be highly cytocompatible (cell viability $>95 \%$ ) and no significant difference with respect to cytocompatibility was observed for the hydrogel blend in comparison to plain alginate solution. Plain A-PPhenEtOx-A was not tested for cytocompatibility during bioprinting. On the one hand, it is known from the literature that very similar polymers do not show cytotoxic properties up to a concentration of $100 \mathrm{~g} / \mathrm{L} .{ }^{39,42}$ On the other hand, addition of $1 \mathrm{wt} \%$ alginate should not have any significant effect in terms of cytocompatibility.

\section{CONCLUSIONS}

Here, a new ABA-type amphiphile based on poly(2-oxazoline)s with an aromatic hydrophobic central block of poly(2phenethyl-2-oxazoline) B was described. Above the CMC, small spherical nanoscale micelles were confirmed. At or above $20 \mathrm{wt} \%$, an inverse and fast sol/gel transition was observed upon cooling, due to densely packed micelles and investigated via temperature-dependent ${ }^{1} \mathrm{H}$ NMR and SAXS experiments. Favorable rheological properties, such as pronounced viscoelastic solid-like properties, shear-thinning, and structure recovery properties led to very good $3 \mathrm{D}$-printability of the investigated hydrogel. In a hydrogel blend comprising the readily printable POx-based polymer and the biopolymer alginate, stable constructs were obtained. First cytocompatible bioprinting at approximately $10{ }^{\circ} \mathrm{C}$ for a A-PPhenEtOx-A/ alginate hybrid system concluded the study and opens a wide range of different applications in the field of biofabrication.

\section{ASSOCIATED CONTENT}

\section{Supporting Information}

The Supporting Information is available free of charge at https://pubs.acs.org/doi/10.1021/acs.biomac.1c00427.

Detailed description of monomer and polymer synthesis and characterization and an overview and detailed description of SAXS analysis (PDF)

Additional movie of the extrusion printing process (MP4)

\section{AUTHOR INFORMATION}

\section{Corresponding Author}

Robert Luxenhofer - Functional Polymer Materials, Chair for Advanced Materials Synthesis, Institute for Functional Materials and Biofabrication, Department of Chemistry and Pharmacy, Julius-Maximilians-University Würzburg, Würzburg 97070, Germany; Soft Matter Chemistry, Department of Chemistry and Helsinki Institute of Sustainability Science, Faculty of Science, University of Helsinki, Helsinki 00014, Finland; 이이.org/0000-00015567-7404; Email: robert.luxenhofer@helsinki.fi

\section{Authors}

Lukas Hahn - Functional Polymer Materials, Chair for Advanced Materials Synthesis, Institute for Functional Materials and Biofabrication, Department of Chemistry and Pharmacy, Julius-Maximilians-University Würzburg, Würzburg 97070, Germany

Emine Karakaya - Institute of Biomaterials, Friedrich Alexander University of Erlangen-Nürnberg, Erlangen 91058, Germany

Theresa Zorn - Institute of Organic Chemistry, JuliusMaximilians-University Würzburg, Würzburg 97074, Germany

Benedikt Sochor - Chair for X-Ray Microscopy, JuliusMaximilians-University Würzburg, Würzburg 97074, Germany; 이이.org/0000-0002-5772-8065

Matthias Maier - Functional Polymer Materials, Chair for Advanced Materials Synthesis, Institute for Functional Materials and Biofabrication, Department of Chemistry and Pharmacy, Julius-Maximilians-University Würzburg, Würzburg 97070, Germany

Philipp Stahlhut - Department for Functional Materials in Medicine and Dentistry, Julius-Maximilians-University Würzburg, Würzburg 97070, Germany

Stefan Forster - Functional Polymer Materials, Chair for Advanced Materials Synthesis, Institute for Functional Materials and Biofabrication, Department of Chemistry and Pharmacy, Julius-Maximilians-University Würzburg, Würzburg 97070, Germany

Karl Fischer - Department of Chemistry, Johannes Gutenberg University Mainz, Mainz 55128, Germany

Sebastian Seiffert - Department of Chemistry, Johannes Gutenberg University Mainz, Mainz 55128, Germany; () orcid.org/0000-0002-5152-1207

Ann-Christin Pöppler - Institute of Organic Chemistry, JuliusMaximilians-University Würzburg, Würzburg 97074, Germany; 이이.org/0000-0002-0624-1708

Rainer Detsch - Institute of Biomaterials, Friedrich Alexander University of Erlangen-Nürnberg, Erlangen 91058, Germany

Complete contact information is available at:

https://pubs.acs.org/10.1021/acs.biomac.1c00427 


\section{Notes}

The authors declare the following competing financial interest(s): L.H. and R.L. are listed as inventors on a patent pertinent to some materials in the present work.

\section{ACKNOWLEDGMENTS}

The authors gratefully acknowledge support by the Deutsche Forschungsgemeinschaft (DFG, German Research Foundation)-project number 326998133-TRR225 (subprojects A03, B06). Furthermore, we thank the Deutsche Forschungsgemeinschaft for funding the crossbeam scanning electron microscope Zeiss CB 340 (INST 105022/58-1 FUGG) within the DFG State Major Instrumentation Programme. In addition, light scattering experiments were possible through support of the Deutsche Forschungsgemeinschaft (INST 93/774-1 FUGG). We gratefully acknowledge access to electron microscopy facilities provided by Prof. Bettina Böttcher at the Rudolf Virchow Center, Julius-Maximilians-University Würzburg.

\section{REFERENCES}

(1) Zhang, Q.; Weber, C.; Schubert, U. S.; Hoogenboom, R. Thermoresponsive Polymers with Lower Critical Solution Temperature: From Fundamental Aspects and Measuring Techniques to Recommended Turbidimetry Conditions. Mater. Horiz. 2017, 4, 109116.

(2) Seuring, J.; Agarwal, S. Non-Ionic Homo- and Copolymers with $\mathrm{H}$-Donor and $\mathrm{H}$-Acceptor Units with an UCST in Water. Macromol. Chem. Phys. 2010, 211, 2109-2117.

(3) Seuring, J.; Agarwal, S. Polymers with Upper Critical Solution Temperature in Aqueous Solution. ACS Macro Lett. 2012, 33, 18981920.

(4) Shimada, N.; Ino, H.; Maie, K.; Nakayama, M.; Kano, A.; Maruyama, A. Ureido-Derivatized Polymers Based on Both Poly(Allylurea) and Poly(L-Citrulline) Exhibit UCST-Type Phase Transition Behavior under Physiologically Relevant Conditions. Biomacromolecules 2011, 12, 3418-3422.

(5) Meiswinkel, G.; Ritter, H. A New Type of Thermoresponsive Copolymer with UCST-Type Transitions in Water: Poly(N-Vinylimidazole-co-1-Vinyl-2-(Hydroxymethyl)imidazole). Macromol. Rapid Commun. 2013, 34, 1026-1031.

(6) Aseyev, V.; Tenhu, H.; Winnik, F., Non-Ionic Thermoresponsive Polymers in Water. Springer, Berlin, Heidelberg. 2010, 242, 29-89.

(7) Niskanen, J.; Tenhu, H. How to Manipulate the Upper Critical Solution Temperature (UCST)? Polym. Chem. 2017, 8, 220-232.

(8) Seuring, J.; Bayer, F. M.; Huber, K.; Agarwal, S. Upper Critical Solution Temperature of Poly(N-acryloyl glycinamide) in Water: A Concealed Property. Macromolecules 2012, 45, 374-384.

(9) Zhao, C.; Dolmans, L.; Zhu, X. X. Thermoresponsive Behavior of Poly(acrylic acid-co-acrylonitrile) with a UCST. Macromolecules 2019, 52, 4441-4446.

(10) Plunkett, K. N.; Zhu, X.; Moore, J. S.; Leckband, D. E. PNIPAM Chain Collapse Depends on the Molecular Weight and Grafting Density. Langmuir 2006, 22, 4259-4266.

(11) Gupta, N. R.; Torris, A. T. A.; Wadgaonkar, P. P.; Rajamohanan, P. R.; Ducouret, G.; Hourdet, D.; Creton, C.; Badiger, M. V. Synthesis and Characterization of PEPO Grafted Carboxymethyl Guar and Carboxymethyl Tamarind as New Thermo-Associating Polymers. Carbohydr. Polym. 2015, 117, 331-338.

(12) Karakasyan, C.; Lack, S.; Brunel, F.; Maingault, P.; Hourdet, D. Synthesis and Rheological Properties of Responsive Thickeners Based on Polysaccharide Architectures. Biomacromolecules 2008, 9, 24192429.

(13) Bokias, G.; Mylonas, Y.; Staikos, G.; Bumbu, G. G.; Vasile, C. Synthesis and Aqueous Solution Properties of Novel Thermoresponsive Graft Copolymers Based on a Carboxymethylcellulose Backbone. Macromolecules 2001, 34, 4958-4964.
(14) Koonar, I.; Zhou, C.; Hillmyer, M. A.; Lodge, T. P.; Siegel, R. A. ABC Triblock Terpolymers Exhibiting Both Temperature- and $\mathrm{pH}$ Sensitive Micellar Aggregation and Gelation in Aqueous Solution. Langmuir 2012, 28, 17785-17794.

(15) Taribagil, R. R.; Hillmyer, M. A.; Lodge, T. P. Hydrogels from ABA and ABC Triblock Polymers. Macromolecules 2010, 43, 53965404.

(16) Niu, H.; Wang, F.; Weiss, R. A. Hydrophobic/Hydrophilic Triblock Copolymers: Synthesis and Properties of Physically CrossLinked Hydrogels. Macromolecules 2015, 48, 645-654.

(17) Tuncaboylu, D. C.; Argun, A.; Sahin, M.; Sari, M.; Okay, O. Structure Optimization of Self-Healing Hydrogels Formed Via Hydrophobic Interactions. Polymer 2012, 53, 5513-5522.

(18) Djabourov, M.; Leblond, J.; Papon, P. Gelation of Aqueous Gelatin Solutions. II. Rheology of the Sol-Gel Transition. J. Phys. France 1988, 49, 333-343.

(19) Birch, N. P.; Barney, L. E.; Pandres, E.; Peyton, S. R.; Schiffman, J. D. Thermal-Responsive Behavior of a Cell Compatible Chitosan/ Pectin Hydrogel. Biomacromolecules 2015, 16, 1837-1843.

(20) Yoshioka, H.; Mori, Y.; Tsukikawa, S.; Kubota, S. Thermoreversible Gelation on Cooling and on Heating of an Aqueous GelatinPoly(N-isopropylacrylamide) Conjugate. Polym. Adv. Technol. 1998, 9, $155-158$.

(21) Fu, W.; Zhao, B. Thermoreversible Physically Crosslinked Hydrogels from UCST-Type Thermosensitive ABA Linear Triblock Copolymers. Polym. Chem. 2016, 7, 6980-6991.

(22) Parmar, I. A.; Shedge, A. S.; Badiger, M. V.; Wadgaonkar, P. P.; Lele, A. K. Thermo-Reversible Sol-Gel Transition of Aqueous Solutions of Patchy Polymers. RSC Adv. 2017, 7, 5101-5110.

(23) Zheng, J.; Xiao, P.; Le, X.; Lu, W.; Théato, P.; Ma, C.; Du, B.; Zhang, J.; Huang, Y.; Chen, T. Mimosa Inspired Bilayer Hydrogel Actuator Functioning in Multi-Environments. J. Mater. Chem. C 2018, $6,1320-1327$

(24) Hua, L.; Xie, M.; Jian, Y.; Wu, B.; Chen, C.; Zhao, C. MultipleResponsive and Amphibious Hydrogel Actuator Based on Asymmetric UCST-Type Volume Phase Transition. ACS Appl. Mater. Interfaces 2019, 11, 43641-43648.

(25) Groll, J.; Boland, T.; Blunk, T.; Burdick, J. A.; Cho, D.; Dalton, P. D.; Derby, B.; Frogacs, G.; Li, Q.; Mironov, V. A.; Moroni, L.; Nakamura, M.; Shu, W.; Takeuchi, S.; Vozzi, G.; Woodfield, T. B. F.; $\mathrm{Xu}, \mathrm{T}$.; Yoo, J. J.; Malda, J. Biofabrication: Reappraising the Definition of an Evolving Field. Biofabrication 2016, 8, No. 13001.

(26) Groll, J.; Burdick, J. A.; Cho, D. W.; Derby, B.; Gelinsky, M.; Heilshorn, S. C.; Jüngst, T.; Malda, J.; Mironov, V. A.; Nakayama, K.; Ovsianikov, A.; Sun, W.; Takeuchi, S.; Yoo, J. J.; Woodfield, T. B. F. A Definition of Bioinks and Their Distinction from Biomaterial Inks. Biofabrication 2019, 11, No. 013001.

(27) Chimene, D.; Kaunas, R.; Gaharwar, A. K. Hydrogel Bioink Reinforcement for Additive Manufacturing: A Focused Review of Emerging Strategies. Adv. Mater. 2020, 32, No. 1902026.

(28) Valot, L.; Martinez, J.; Mehdi, A.; Subra, G. Chemical Insights into Bioinks for 3D Printing. Chem. Soc. Rev. 2019, 48, 4049-4086.

(29) Glassner, M.; Vergaelen, M.; Hoogenboom, R. Poly(2oxazoline)s: A Comprehensive Overview of Polymer Structures and Their Physical Properties. Polym. Int. 2018, 67, 32-45.

(30) Lorson, T.; Lübtow, M. M.; Wegener, E.; Haider, M. S.; Borova, S.; Nahm, D.; Jordan, R.; Sokolski-Papkov, M.; Kabanov, A. V.; Luxenhofer, R. Poly(2-oxazoline)s Based Biomaterials: A Comprehensive and Critical Update. Biomaterials 2018, 178, 204-280.

(31) Luxenhofer, R.; Jordan, R. Poly(2-oxazoline)s (POx) in Biomedical Applications. Mater. Matters 2013, 8, 70-73.

(32) Dargaville, T. R.; Park, J. R.; Hoogenboom, R. Poly(2-oxazoline) Hydrogels: State-of-the-Art and Emerging Applications. Macromol. Biosci. 2018, 18, No. e1800070.

(33) Harris, J. M.; Bentley, M. D.; Moreadith, R. W.; Viegas, T. X.; Fang, Z.; Yoon, K.; Weimer, R.; Dizman, B.; Nordstierna, L. Tuning Drug Release from Polyoxazoline-Drug Conjugates. Eur. Polym. J. 2019, 120, No. 109241. 
(34) Moreadith, R. W.; Viegas, T. X.; Bentley, M. D.; Harris, J. M.; Fang, Z.; Yoon, K.; Dizman, B.; Weimer, R.; Rae, B. P.; Li, X.; Rader, C.; Standaert, D.; Olanow, W. Clinical Development of a Poly(2oxazoline) (POz) Polymer Therapeutic for the Treatment of Parkinson's Disease - Proof of Concept of POz as a Versatile Polymer Platform for Drug Development in Multiple Therapeutic Indications. Eur. Polym. J. 2017, 88, 524-552.

(35) Sedlacek, O.; Hoogenboom, R. Drug Delivery Systems Based on Poly(2-oxazoline)s and Poly(2-oxazine)s. Adv. Ther. 2020, 3, No. 1900168.

(36) Lorson, T.; Jaksch, S.; Lübtow, M. M.; Jüngst, T.; Groll, J.; Lühmann, T.; Luxenhofer, R. A Thermogelling Supramolecular Hydrogel with Sponge-Like Morphology as a Cytocompatible Bioink. Biomacromolecules 2017, 18, 2161-2171.

(37) Monnery, B. D.; Hoogenboom, R. Thermoresponsive Hydrogels Formed by Poly(2-oxazoline) Triblock Copolymers. Polym. Chem. 2019, 10, 3480-3487.

(38) Lübtow, M. M.; Mrlik, M.; Hahn, L.; Altmann, A.; Beudert, M.; Lühmann, T.; Luxenhofer, R. Temperature-Dependent Rheological and Viscoelastic Investigation of a Poly(2-methyl-2-oxazoline)-bpoly(2-iso-butyl-2-oxazoline)-b-poly(2-methyl-2-oxazoline)-Based Thermogelling Hydrogel. J. Funct. Biomater. 2019, 10, 36.

(39) Hahn, L.; Maier, M.; Stahlhut, P.; Beudert, M.; Flegler, V.; Forster, S.; Altmann, A.; Töppke, F.; Fischer, K.; Seiffert, S.; Böttcher, B.; Lühmann, T.; Luxenhofer, R. Inverse Thermogelation of Aqueous Triblock Copolymer Solutions into Macroporous Shear-Thinning 3D Printable Inks. ACS Appl. Mater. Interfaces 2020, 12, 12445-12456.

(40) Hahn, L.; Zorn, T.; Kehrein, J.; Kielholz, T.; Sochor, B.; Lisitsyna, E. S.; Durandin, N.; Laaksonen, T.; Aseyev, V.; Sotriffer, C.; Windbergs, M.; Pöppler, A. C.; Luxenhofer, R., Unravelling a Novel Sol-gel Transition Mechanism in Polymer Self-assemblies: An Order-order Transition Based on Specific Molecular Interactions Between Hydrophilic and Hydrophobic Polymer Blocks, 2021. DOI: 10.26434/chemrxiv.14362892.v1.

(41) Witte, H.; Seeliger, W. Simple Synthesis of 2-Substituted 2Oxazolines and 5,6-Dihydro-4h-1,3-Oxazines. Angew. Chem., Int. Ed. Engl. 1972, 11, 287-288.

(42) Hahn, L.; Lübtow, M. M.; Lorson, T.; Schmitt, F.; AppeltMenzel, A.; Schobert, R.; Luxenhofer, R. Investigating the Influence of Aromatic Moieties on the Formulation of Hydrophobic Natural Products and Drugs in Poly(2-oxazoline)-Based Amphiphiles. Biomacromolecules 2018, 19, 3119-3128.

(43) Lübtow, M. M.; Hahn, L.; Haider, M. S.; Luxenhofer, R. Drug Specificity, Synergy and Antagonism in Ultrahigh Capacity Poly(2oxazoline)/Poly(2-oxazine) Based Formulations. J. Am. Chem. Soc. 2017, 139, 10980-10983.

(44) Konefal, R.; Spěváček, J.; Černoch, P. Thermoresponsive Poly(2oxazoline) Homopolymers and Copolymers in Aqueous Solutions Studied by NMR Spectroscopy and Dynamic Light Scattering. Eur. Polym. J. 2018, 100, 241-252.

(45) Dreiss, C. A.; Jack, K. S.; Parker, A. P. On the Absolute Calibration of Bench-Top Small-Angle X-Ray Scattering Instruments: A Comparison of Different Standard Methods. J. Appl. Crystallogr. 2006, 39, 32-38.

(46) Zhang, F.; Ilavsky, J.; Long, G. G.; Quintana, J. P. G.; Allen, A. J.; Jemian, P. R. Glassy Carbon as an Absolute Intensity Calibration Standard for Small-Angle Scattering. Metall. Mater. Trans. A 2010, 41, $1151-1158$.

(47) Pauw, B. R.; Smith, A. J.; Snow, T.; Terrill, N. J.; Thünemann, A. F. The Modular Small-Angle X-Ray Scattering Data Correction Sequence. J. Appl. Crystallogr. 2017, 50, 1800-1811.

(48) Ribeiro, A.; Blokzijl, M. M.; Levato, R.; Visser, C. W.; Castilho, M.; Hennink, W. E.; Vermonden, T.; Malda, J. Assessing Bioink Shape Fidelity to Aid Material Development in 3D Bioprinting. Biofabrication 2018, 10, No. 014102.

(49) Luxenhofer, R.; Schulz, A.; Roques, C.; Li, S.; Bronich, T. K.; Batrakova, E. V.; Jordan, R.; Kabanov, A. V. Doubly Amphiphilic Poly(2-oxazoline)s as High-Capacity Delivery Systems for Hydrophobic Drugs. Biomaterials 2010, 31, 4972-4979.
(50) Seo, Y.; Schulz, A.; Han, Y.; He, Z.; Bludau, H.; Wan, X.; Tong, J.; Bronich, T. K.; Sokolsky, M.; Luxenhofer, R.; Jordan, R.; Kabanov, A. V. Poly(2-oxazoline) Block Copolymer Based Formulations of Taxanes: Effect of Copolymer and Drug Structure, Concentration, and Environmental Factors. Polym. Adv. Technol. 2015, 26, 837-850.

(51) Sood, N.; Bhardwaj, A.; Mehta, S.; Mehta, A. Stimuli-Responsive Hydrogels in Drug Delivery and Tissue Engineering. Drug Delivery 2016, 23, 748-770.

(52) Malda, J.; Visser, J.; Melchels, F. P.; Jüngst, T.; Hennink, W. E.; Dhert, W. J. A.; Groll, J.; Hutmacher, D. W. $25^{\text {th }}$ Anniversary Article: Engineering Hydrogels for Biofabrication. Adv. Mater. 2013, 25, 50115028.

(53) Naomi, P.; Willi, S.; Thomas, B.; Ferry, M.; Jürgen, G.; Tomasz, J. Proposal to Assess Printability of Bioinks for Extrusion-Based Bioprinting and Evaluation of Rheological Properties Governing Bioprintability. Biofabrication 2017, 9, No. 044107.

(54) Hazur, J.; Detsch, R.; Karakaya, E.; Kaschta, J.; Teßmar, J.; Schneidereit, D.; Friedrich, O.; Schubert, D. W.; Boccaccini, A. R. Improving Alginate Printability for Biofabrication: Establishment of a Universal and Homogeneous Pre-Crosslinking Technique. Biofabrication 2020, 12, No. 045004. 